\title{
Quantitative Doppler tissue imaging as a correlate of left ventricular contractility
}

\author{
David S. Bach \\ Department of Internal Medicine, Division of Cardiology University of Michigan, Ann Arbor, MI 48109, USA
}

Accepted 12 April 1996

Key words: echocardiography/Doppler, left ventricular function, myocardial contractility

\begin{abstract}
Doppler tissue imaging is a new noninvasive imaging modality that allows quantitation of the low intensity, high amplitude Doppler shifts in the range of myocardial tissue motion. This study was performed to test the hypothesis that Doppler tissue imaging may provide unique information reflecting left ventricular systolic function, and to test the relationship between myocardial tissue velocity and noninvasive measures of ventricular contractility. Nine patients with mild or moderate mitral insufficiency and no regional wall motion abnormality were studied during dobutamine stress echocardiography. Left ventricular ejection fraction and peak systolic velocity of the sub- endocardial left ventricular posterior wall were quantified at baseline and at peak stress and compared with estimated peak $\mathrm{dP} / \mathrm{dt}$. During dobutamine infusion, ejection fraction increased from $41.7 \pm 22.2$ (range 14 to 70 ) $\%$ to $56.6 \pm 27.9$ (range 17 to 84$) \%(p=0.001$ ), peak systolic velocity increased from $22.7 \pm 4.2$ (range 18 to 28 ) $\mathrm{mm} / \mathrm{sec}$ to $35.3 \pm 10.1$ (range 20 to 47$) \mathrm{mm} / \mathrm{sec}(\mathrm{p}=0.004$ ), and $\mathrm{dP} / \mathrm{dt}$ increased from $1050 \pm 322$ (range 613 to 1574) $\mathrm{mm} \mathrm{Hg} / \mathrm{sec}$ to $1766 \pm 768$ (range 936 to 3000$) \mathrm{mm} \mathrm{Hg} / \mathrm{sec}(\mathrm{p}=0.01$ ). Although there were good correlations between left ventricular $\mathrm{dP} / \mathrm{dt}$ and both ejection fraction $(\mathrm{R}=0.75)$ and peak systolic velocity $(\mathrm{R}=0.81)$, the correlation between change in $\mathrm{dP} / \mathrm{dt}$ and change in myocardial velocity $(\mathrm{R}=0.75)$ was better than that between change in $\mathrm{dP} / \mathrm{dt}$ and change in ejection fraction $(\mathrm{R}=0.36)$. These data support the hypothesis that myocardial velocity determined with Doppler tissue imaging reflects myocardial contractility, and that catecholamine-induced alteration in contractility is better reflected by changes in myocardial velocity than by changes in ejection fraction.
\end{abstract}

\section{Introduction}

Regional myocardial contractility is not readily assessed using noninvasive techniques. Measures of left ventricular function such as ejection fraction and Doppler left ventricular outflow velocities are sensitive to alterations in loading conditions. Further, both of these parameters reflect global ventricular function; assessment of regional myocardial function is more difficult to quantify.

Doppler tissue imaging is a new noninvasive imaging modality in which specialized imaging software is used to interrogate the low velocity, high intensity Doppler signals in the range of myocardial tissue motion [1-5]. Although developed as an adjunct for qualitative wall motion analysis [1-3], quantitative velocity data can be independently assessed [2-
5]. Myocardial velocities have been previously validated against tissue velocities derived using $\mathrm{M}$-mode echocardiography $[3,4]$, quantified among normal volunteers [2-4], and found to be significantly reduced among patients with cardiomyopathy $[2,3]$. Because Doppler tissue imaging can directly interrogate the velocity of systolic myocardial thickening, it may provide unique information reflecting regional ventricular systolic function. This study was performed to test this hypothesis, and to test whether myocardial velocities determined using Doppler tissue imaging reflect changes in myocardial contractility better than existing noninvasive measures. To this end, peak systolic myocardial velocity derived using quantitative Doppler tissue imaging and left ventricular ejection fraction were both compared to noninvasively estimated left ventricular $\mathrm{dP} / \mathrm{dt}$ under varying inotropic states. 


\section{Methods}

\section{Study population}

The study population comprised nine patients with mild or moderate mitral insufficiency in non-eccentric jets, and no regional wall motion abnormality referred to the Echocardiography Laboratory at the University of Michigan for dobutamine stress echocardiography. There were three men and six women age $64 \pm 13$ (range 35 to 82 ) years. Five subjects had normal resting left ventricular function; the remaining four subjects had cardiomyopathy of varying severity but with no regional abnormality in wall motion. Resting left ventricular ejection fraction was 14 to $70 \%$. Dobutamine stress echocardiography was performed as previously described [6]. In brief, dobutamine (Dobutrex, Eli Lilly, Indianapolis, IN) was infused in four threeminute stages at doses of $10,20,30$ and $40 \mathrm{mcg} / \mathrm{kg} / \mathrm{min}$. Dobutamine infusion was terminated when heart rate reached $85 \%$ of age-predicted maximum or with completion of the infusion protocol, whichever came first. Atropine was not administered.

\section{EcholDoppler imaging}

Patients were studied in a left lateral decubitous position using an Acuson 128XP/10 (Acuson Corp., Mountain View, CA) with specialized software for the interrogation of low velocity Doppler signals $[2,4)$. All quantitative echo/Doppler imaging was performed from standard transthoracic windows. Both at baseline and at peak dobutamine infusion, apical 4-chamber and 2- chamber images were recorded on standard VHS videotape for subsequent off-line calculation of left ventricular ejection fraction. Immediately thereafter, mitral insufficiency was interrogated using continuous wave Doppler from an apical window, with spectral signals recorded on videotape for subsequent calculation of estimated left ventricular $\mathrm{dP} / \mathrm{dt}$. Doppler tissue imaging was then performed from a parasternal shortaxis view. At both stages, imaging for ejection fraction, $\mathrm{dP} / \mathrm{dt}$ and Doppler tissue velocity was complete within one minute.

\section{Doppler tissue imaging}

Doppler tissue imaging was limited to the posterior wall myocardium, such that systolic thickening in the region of interest occurred parallel to or within $15^{\circ}$ on either side of the interrogating Doppler signal. Doppler tissue interrogation was further limited to a specific region of interest, permitting Doppler sampling rates of 20 to $40 \mathrm{~Hz}$. Gain settings and filters were adjusted to optimize color saturation. Doppler Nyquist limits were adjusted to eliminate color aliasing. Both at baseline and during peak dobutamine infusion, myocardial velocities of the subendocardial posterior wall were quantified on-line throughout at least three cardiac cycles. Maximal systolic velocities were determined for each cardiac cycle, and peak systolic velocities for at least three cardiac cycles were averaged during each phase.

\section{Quantitative echo/Doppler}

Off-line analysis of left ventricular ejection fraction and left ventricular $\mathrm{dP} / \mathrm{dt}$ were performed using a digital work station (Tomtec, Broomfield, CO). Left ventricular volumes and ejection fraction were calculated using Simpson's method with bi-apical views [7]. Left ventricular peak $\mathrm{dP} / \mathrm{dt}$ was estimated using a previously validated method based upon the rate of early acceleration of mitral insufficiency on continuous wave Doppler [8, 9]. In brief, mitral insufficiency was interrogated along an axis parallel to the direction of flow using continuous wave Doppler from an apical window. Using an expanded scale and a rapid sweep speed, elapsed time $(\Delta t)$ was measured between mitral insufficiency velocities of 1.0 and $3.0 \mathrm{~m} / \mathrm{s}$. Based on the modified Bernoulli equation $\left(P=4 \times V^{2}\right)$, these velocities correspond to pressure differences between chambers of 4 and $36 \mathrm{~mm} \mathrm{Hg}$, respectively. The change in pressure $(\Delta \mathrm{P})$ between these two time points was calculated as $\Delta \mathrm{P}=\left[\mathrm{P}_{2}-\mathrm{P}_{1}\right]=\left[\left(4 \times 3^{2}\right)-\left(4 \times 1^{2}\right)\right]=$ $[36-4]=32 \mathrm{~mm} \mathrm{Hg}$. Peak $\mathrm{dP} / \mathrm{dt}$ was taken as the ratio of change in pressure $(32 \mathrm{~mm} \mathrm{Hg})$ to change in time $(\Delta t)$ in seconds $(\Delta P / \Delta t)$.

\section{Statistics}

All data are presented as mean \pm 1 SD. Comparison between ejection fraction, myocardial velocity and $\mathrm{dP} / \mathrm{dt}$ at baseline and during peak dobutamine infusion were made using paired t-tests. Analyses of correlation between ejection fraction or myocardial velocity and $\mathrm{dP} / \mathrm{dt}$, as well as correlation between change in ejection fraction or change in velocity and change in $\mathrm{dP} / \mathrm{dt}$ were made using Pearson correlation coefficients. Differences were considered significant at a value of $p<$ 0.05 (two-sided). 
Table 1. Change in left ventricular ejection fraction, myocardial velocity and estimated $\mathrm{dP} / \mathrm{dt}$ during dobutamine infusion.

\begin{tabular}{lcccc}
\hline & Baseline & Dobutamine & $\Delta$ & $\mathrm{p}$ \\
\hline Ejection fraction (\%) & $41.7 \pm 22.2$ & $56.6 \pm 27.9$ & $14.9 \pm 8.3$ & 0.001 \\
(range) & $(14$ to 70$)$ & $(17$ to 84$)$ & $(0$ to 26$)$ & \\
Velocity $(\mathrm{mm} / \mathrm{sec})$ & $22.7 \pm 4.2$ & $35.3 \pm 10.1$ & $12.6 \pm 8.7$ & 0.004 \\
(range) & $(18$ to 28$)$ & $(20$ to 47$)$ & $(-5$ to 21$)$ & \\
dP/dt (mm Hg/sec) & $1050 \pm 322$ & $1766 \pm 768$ & $716 \pm 622$ & 0.01 \\
(range) & $(613$ to 1574$)$ & $(936$ to 3000$)$ & $(-114$ to 1716$)$ & \\
\hline
\end{tabular}
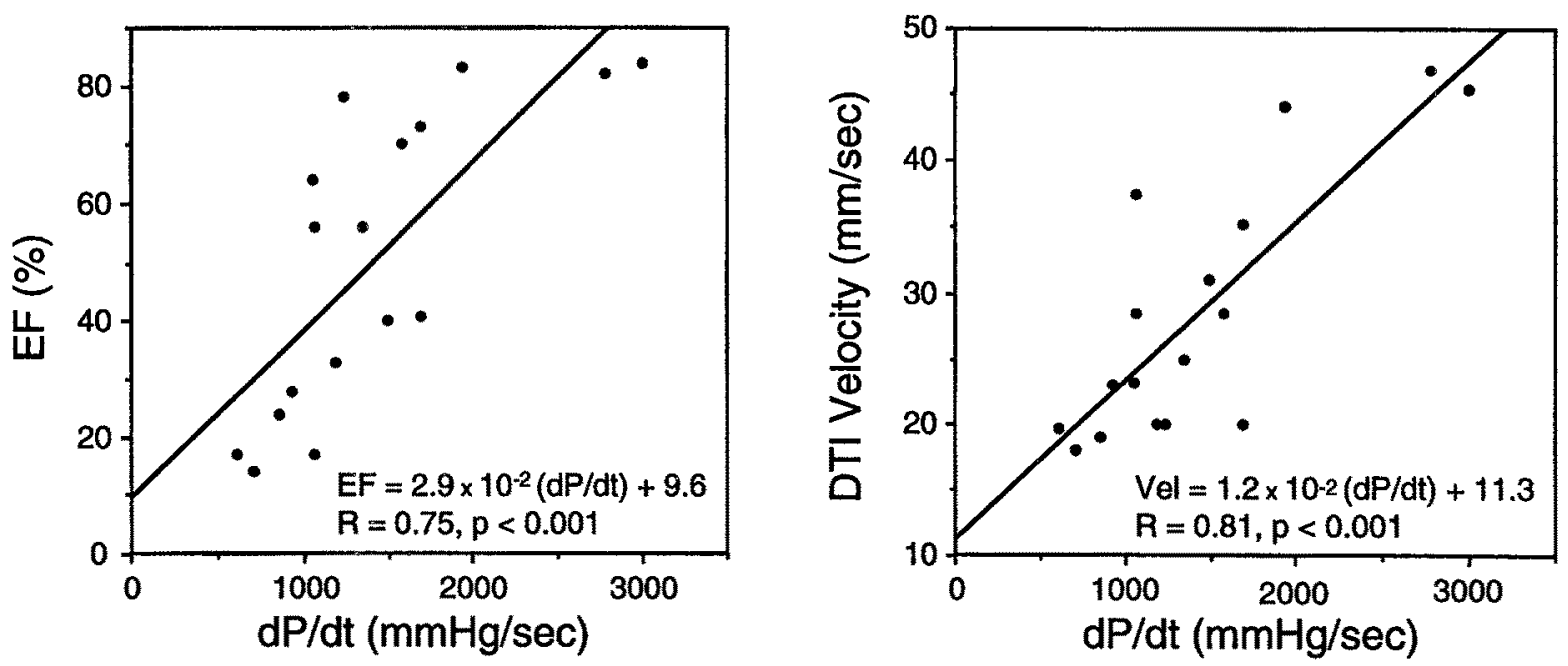

Figure 1. Relationships between left ventricular ejection fraction and estimated dP/dt (left) and between peak systolic myocardial velocity from Doppler tissue imaging and $\mathrm{dP} / \mathrm{dt}$ (right) for all subjects at both baseline and during dobutamine infusion.

\section{Results}

\section{Dobutamine infusion}

All subjects received a peak rate of dobutamine infusion of at least $30 \mathrm{mcg} / \mathrm{kg} / \mathrm{min}$ (mean $34 \pm 7$ $\mathrm{mcg} / \mathrm{kg} / \mathrm{min}$ ). Heart rate increased from $73 \pm 20$ (range 50 to 106) beats/min to $104 \pm 24$ (range 66 to 135 ) beats $/ \mathrm{min}$. Systolic blood pressure remained relatively stable between baseline ( $149 \pm 35$, range 99 to 195 $\mathrm{mm} \mathrm{Hg}$ ) and peak infusion (152 \pm 42 , range 95 to 217 $\mathrm{mm} \mathrm{Hg}$ ). Left ventricular ejection fraction, peak systolic myocardial velocity from Doppler tissue imaging and estimated peak $\mathrm{dP} / \mathrm{dt}$ at baseline and during peak dobutamine infusion are summarized in Table 1.

\section{Relationship with $d P / d t$}

The relationships between left ventricular ejection fraction and $\mathrm{dP} / \mathrm{dt}$ and between peak systolic myocar- dial velocity derived using Doppler tissue imaging and $\mathrm{dP} / \mathrm{dt}$ for all subjects at both baseline and during dobutamine infusion are shown in Figure 1. There were good correlations between left ventricular $\mathrm{dP} / \mathrm{dt}$ and both ejection fraction and peak systolic velocity, with $\mathrm{R}$ values of 0.75 and 0.81 , respectively. The relationships between change in $\mathrm{dP} / \mathrm{dt}$ during dobutamine infusion and change in ejection fraction and change in peak systolic myocardial velocity are shown in Figure 2. Although there was poor correlation between change in ejection fraction and change in $\mathrm{dP} / \mathrm{dt}(\mathrm{R}=0.36)$, there was a good correlation between change in myocardial velocity and change in $\mathrm{dP} / \mathrm{dt}(\mathrm{R}=0.75)$. 

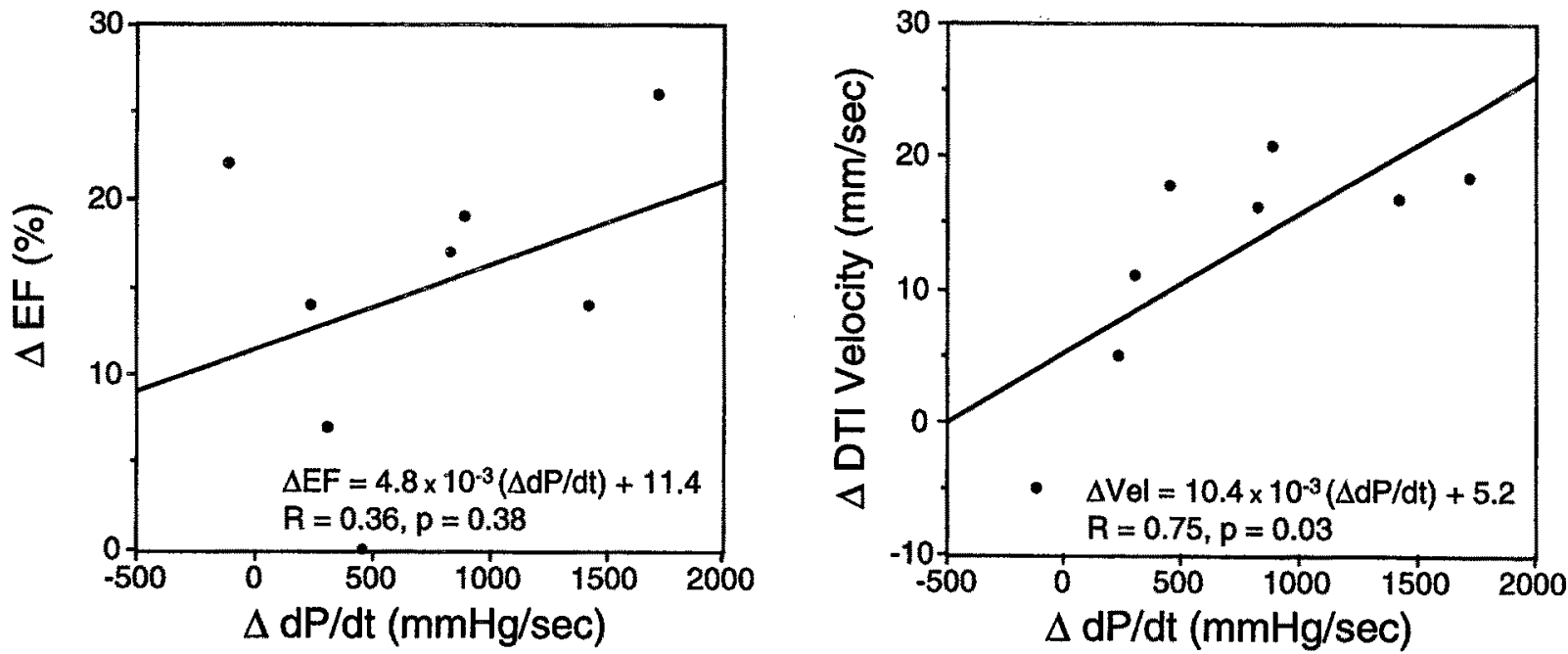

Figure 2. Relationships between change in estimated $\mathrm{dP} / \mathrm{dt}$ during dobutamine infusion and change in ejection fraction (left) and change in peak systolic myocardial velocity (right).

\section{Discussion}

\section{Measures of systolic function}

Noninvasive assessment of systolic ventricular function has been previously limited to parameters reflecting global performance such as fractional shortening, ejection fraction and Doppler indices of left ventricular outflow. Although these are adequate for defining global function, regional systolic performance cannot be readily assessed. M-mode analysis of percent myocardial thickening can be used to assess regional systolic performance, but is limited to regions in which the direction of systolic thickening occurs parallel to the interrogating M-mode signal. Doppler tissue imaging is a new noninvasive imaging technique that takes advantage of the unique echocardiographic and Doppler properties of myocardium and myocardial motion [1-5]. Unlike motion associated with the blood pool, myocardial velocities are of high intensity and low velocity. By selectively targeting these low velocity, high amplitude Doppler shifts, Doppler tissue imaging can accurately and reliably interrogate regional myocardial systolic velocities [2-4]. Doppler tissue imaging can be used to interrogate velocities throughout various regions of myocardium [2-4]. Because the direction of myocardial thickening parallels that of the interrogating Doppler signal in only limited regions, the posterior wall was targeted for analysis in the present study. However, algorithms have been developed [5] that allow assessment and comparison of velocities throughout the myocardium using Doppler tissue imaging.

\section{Myocardial contractility}

In the present study, peak systolic myocardial velocities were found to correlate with noninvasively-estimated left ventricular peak $\mathrm{dP} / \mathrm{dt}$. The correlation between myocardial velocity and $\mathrm{dP} / \mathrm{dt}$ was similar to that found between ejection fraction and $\mathrm{dP} / \mathrm{dt}$. However, change in $\mathrm{dP} / \mathrm{dt}$ during inotropic stimulation was much better correlated with change in Doppler tissue velocities than with change in ejection fraction. This suggests that myocardial velocities derived using Doppler tissue imaging may better reflect myocardial contractility than do other noninvasive measures of left ventricular function. Loading conditions were not controlled in the present study. However, preload should not have changed substantially between baseline and peak dobutamine infusion and, although afterload may change during inotropic stimulation, systolic blood pressure remained relatively stable during dobutamine infusion. In this sense, the present study does not address any potential relationship between myocardial tissue velocities and ventricular loading conditions.

\section{Study limitations}

In the present study, measures of myocardial velocity using Doppler tissue imaging were not corrected for incident angle. Errors were minimized by studying 
only the posterior wall myocardium, such that systolic thickening occurred parallel to or within $15^{\circ}$ on either side of the interrogating Doppler signal [4]. Assessment of velocity in other myocardial regions could be performed using an algorithm for correction of incident angle [5]. Left ventricular $\mathrm{dP} / \mathrm{dt}$ was estimated noninvasively from the rate of acceleration of mitral insufficiency. This estimation has been previously shown to correlate with invasive measurements of $\mathrm{dP} / \mathrm{dt}[8,9]$. Because the present study relies on measuring relative differences and relative change in $\mathrm{dP} / \mathrm{dt}$ rather than on its absolute quantitation, the noninvasive estimate should serve as a suitable substitute while avoiding risks associated with invasive testing. Finally, imaging for ejection fraction, continuous wave Doppler and Doppler tissue imaging were not performed simultaneously. Because only Doppler tissue imaging required on-line analysis, images were first rapidly acquired on videotape for subsequent calculation of ejection fraction and $\mathrm{dP} / \mathrm{dt}$, and in all cases imaging was completed within one minute.

\section{Advantages of Doppler tissue imaging}

Doppler tissue imaging is a noninvasive imaging technique that is portable, well tolerated and allows direct, on-line analysis. Because of its ease of use, Doppler tissue imaging can be readily repeated and velocities evaluated for serial change. Doppler tissue imaging has excellent spatial resolution, allowing assessment of regional ventricular performance throughout discrete coronary territories and within distinct myocardial lay. ers between endocardium and epicardium. In addition, the high temporal resolution afforded by Doppler tissue imaging allows assessment of ventricular performance in various phases of the cardiac cycle, including in phases of diastole.

\section{Conclusions}

The results of the present study support the hypothesis that peak systolic myocardial velocity determined with Doppler tissue imaging reflects myocardial contractility measured by peak $\mathrm{dP} / \mathrm{dt}$. Although catecholamine infusion affects left ventricular ejection fraction, catecholamine-induced alteration in myocardial contractility is better reflected by changes in myocardial velocity. Measurement of myocardial velocity using quantitative Doppler tissue imaging may be useful in the noninvasive assessment of ventricu- lar contractility, and may be an effective adjunct to echocardiographic wall motion analysis during catecholamine stress testing.

\section{References}

1. McDicken WN, Sutherland GR, Moran CM, Gordon LN. Colour Doppler velocity imaging of the myocardium. Ultrasound in Med. \& Biol. 1992; 18: 651-654.

2. Sutherland GR, Stewart MJ, Groundstroem KWE, Moran CM, Fleming A, Guell-Peris FJ, et al. Color Doppler myocardial imaging: a new technique for the assessment of myocardial function. J Am Soc Echocardiogr 1994; 7: 441-58.

3. Miyatake K, Yamagishi M, Tanaka N, Uematsu M, Yamazaki $\mathrm{N}$, Mine Yet al. New method for evaluating left ventricular wall motion by color-coded tissue Doppler imaging: in vitro and in vivo studies. J Am Coll Cardiol 1995; 25: 717-24.

4. Donovan CL, Armstrong WF, Bach DS. Quantitative Doppler tissue imaging of the left ventricular myocardium: validation in normal subjects. Am Heart J 1995; 130: 100-4.

5. Uematsu $M$, Miyatake $K$, Tanaka N, Matsuda H, Sano A, Yamazaki N, et al. Myocardial velocity gradient as a new indicator of regional left ventricular contraction: detection by a two-dimensional tissue Doppler imaging technique. J Am Coll Cardiol 1995; 26: 217-23.

6. Bach DS, Armstrong WF. Dobutamine stress echocardiography. Am J Cardiol 1992; 69(20): 90H-96H.

7. Schiller NB, Shah PM, Crawford M, DeMaria A, Devereux $R$, Feigenbaum $H$, Gutgesell $H$, Reichek $N$, Sahn D, Schnittger I, Silverman NH, Tajik AJ. Recommendations for quantitation of the left ventricle by two-dimensional echocardiography. J Am Soc Echo 1989; 2: 358-67.

8. Bargiggia GS, Bertucci C, Recusani F, Raisaro A, de Servi S, Valdez-Cruz LM, et al. A new method for estimating left ventricular $\mathrm{dP} / \mathrm{d}$ by continuous wave Doppler-echocardiography. Circulation 1989; 80: 1287-92.

9. Chen C, Rodriguez L, Guerrero L, Marshall S, Levine RA, Weyman AE, et al. Noninvasive estimation of the instantaneous first derivative of left ventricular pressure using continuouswave Doppler echocardiography. Circulation 1991; 83: 210110.

Address for correspondence: David S. Bach, University of Michigan, UH B1F245 - 0022, 1500 E. Medical Center Drive, Ann Arbor, MI 48109, USA 